\title{
LES VERTUS DE L'ABDICATION : DÉPASSIONNER LA RELATION ENTRE GERMAINE DE STAËL ET NAPOLÉON
}

\section{Stéphanie Genand}

\section{La Fondation Napoléon | « Napoleonica. La Revue »}

$2017 / 3 \mathrm{~N}^{\circ} 30$ | pages 3 à 18

DOI 10.3917/napo.030.0003

\section{Article disponible en ligne à l'adresse :}

https://www.cairn.info/revue-napoleonica-la-revue-2017-3-page-3.htm

Distribution électronique Cairn.info pour La Fondation Napoléon.

(C) La Fondation Napoléon. Tous droits réservés pour tous pays.

La reproduction ou représentation de cet article, notamment par photocopie, n'est autorisée que dans les limites des conditions générales d'utilisation du site ou, le cas échéant, des conditions générales de la licence souscrite par votre établissement. Toute autre reproduction ou représentation, en tout ou partie, sous quelque forme et de quelque manière que ce soit, est interdite sauf accord préalable et écrit de l'éditeur, en dehors des cas prévus par la législation en vigueur en France. Il est précisé que son stockage dans une base de données est également interdit. 


\title{
LES VERTUS DE L'ABDICATION : DÉPASSIONNER LA RELATION ENTRE GERMAINE DE STAËL ET NAPOLÉON
}

\author{
par STÉPHANIE GENAND
}

\section{RÉSUMÉ}

Le titre de cette contribution peut surprendre ou résonner comme une provocation tant il est rarement associé à l'univers de Napoléon. Le terme d'abdication n'y évoque en effet, a priori, que de mauvais souvenirs et l'écho, quelque deux cents plus tard, du renoncement au pouvoir auquel l'Empereur se voit contraint par les forces alliées après la défaite de Waterloo. Cette acception n'est pourtant pas la seule et si le mot d'abdication peut être choisi comme clé programmatique pour réexaminer les relations entre Germaine de Staël et Napoléon, c'est que le terme appartient aussi étroitement au vocabulaire staëlien. Il y revêt, évidemment, un autre sens : non plus historique ni militaire, mais philosophique et plus précisément moral. L’abdication désigne - sous la plume de Mme de Staël, qui la définit dans les Réflexions sur le suicide qu'elle publie en 1812 - l'oubli de soi et le sacrifice de ses intérêts à l'épreuve de la douleur :

"Il n'y a point de doute que nous ne sortions sensiblement meilleurs de l'épreuve de l'adversité, quand nous nous y soumettons avec une fermeté douce. Les plus grandes qualités de l'âme ne se développent que par la souffrance, et ce perfectionnement de nous-mêmes nous rend, après un certain temps, le bonheur. [...] L'existence humaine bien conçue n'est autre chose que l'abdication de la personnalité pour rentrer dans l'ordre universel'."

\section{THE VIRTUES OF ABDICATION (SELF-ANNIHILATION): COOLING DOWN RELATIONS BETWEEN GERMAINE DE STAËL AND NAPOLEON}

Whilst the title here may appear surprising or provocative when applied to the Napoleonic universe - a priori, two hundred years on, the term abdication (self-annihilation) can only evoke bad memories or echoes of the Emperor being forced to step down by the allies after Waterloo - nevertheless, this is not the only resonance possible. The word abdication can also be seen as the programmatic key or decode for relations between Germaine Staël and Napoleon, it being also an essentially Staëlian term. Here, the abdication is not historical or military but rather philosophical and moral. As shown in her Réflexions sur le suicide (which she published in 1812), for Madame de Staël, abdication means self-annihilation and the sacrifice of one's interest when put to the test by pain:

1 Réflexions sur le suicide, 1812, rééd. Oeuvres complètes de Madame de Staël, I-1, 2008, p. 351. 
"It is unquestionable, that whenever we submit to adversity with mild magnanimity, we are refined and improved by the test. The noblest faculties of the soul are developed in suffering, and so salutary is the moral process of amelioration, that after a certain interval, it seldom fails to restore us to tranquility. [...] Human existence, properly understood, is directed to self-annihilation, or the subjection of all petty individual interests, to the principles of eternal truth and justice." 


\title{
LES VERTUS DE L'ABDICATION : DÉPASSIONNER LA RELATION ENTRE GERMAINE DE STAËL ET NAPOLÉON
}

\author{
par STÉPHANIE GENAND \\ Stéphanie Genand est maître de conférences à l'université de Rouen, spécialiste de la \\ littérature du XVIII siècle, présidente de la société des études Staëliennes.
}

Geste d'amputation et d'impersonnalisation, l'abdication prive ainsi le sujet tout en lui promettant, à l'horizon de cette perte consentie, un agrandissement de son âme et de ses facultés, autrement appelé chez Mme de Staël «perfectionnement ». Ce programme exigeant vise aussi bien l'existence de l'individu, invité à tirer paradoxalement profit des épreuves de sa destinée, que la relation à l'autre, $a$ fortiori lorsqu'il s'agit d'un adversaire ou d'un ennemi : assourdir ses passions égoïstes au profit d'une perspective universelle sur l'événement impose en effet de considérer aussi objectivement celui qui fait souffrir et avec qui la relation dépassionnée représente un effort d'autant plus sublime qu' elle a coûté la mise à distance de ses propres douleurs. Les Réflexions sur le suicide explicitent, là encore, la grandeur, voire la nécessité de ce geste :

"La profonde connaissance du cour humain peut seule donner à nos jugements, sur le bonheur et le malheur de ceux qui ne nous ressemblent pas, une équité philosophiquẻ."

Énoncée en 1812, au cœur de l'exil auquel Mme de Staël se voit contrainte par décret le 13 octobre 1803, après la publication de Delphine et de sa préface polémique dédiée à " la France silencieuse ", la formule - « ceux qui ne nous ressemblent pas » - inclut l'ensemble des forces négatives qui oppressent alors l'Europe et dont Napoléon constitue, aux yeux de Mme de Staël, l'incarnation. Faut-il pourtant comprendre que les Réflexions sur le suicide invitent à dépassionner aussi la relation avec l'Empereur ? L'abdication désignerait-elle, dans l'œuvre staëlienne, un geste si courageux qu'il dote la protagoniste elle-même du pouvoir d'envisager de manière neutre le conflit qui l'oppose à l'empereur ?

3 Id., p. 347-348. 


\section{I. À rebours de l'historiographie}

L'hypothèse peut surprendre, et ce d'autant plus qu'elle s'écarte spectaculairement d'une tradition historiographique aussi conséquente que polarisée ${ }^{4}$. Les relations entre Germaine de Staël et Napoléon ont en effet inspiré de très nombreuses études, la plupart historiques, qui ont chacune souligné la violence de la confrontation, éminemment célèbre, entre ces deux figures majeures du tournant des Lumières. Qu'il s'agisse de l'ouvrage de Paul Gautier, Madame de Staël et Napoléon', publié en 1903 et qui tente une reconstitution pionnière de ce combat, ou de l'étude d'Henri Guillemin, Madame de Staël et Napoléon ou Germaine et le caïd ingrat', parue en 1966 et dont le titre explicite la vocation polémique, la virulence dicte le plus souvent, dans cette bibliographie, la reconstitution des faits. Mme de Staël, loin d'incarner une philosophe détachée capable d'abdiquer ses propres passions dans l'événement, y apparaît au contraire comme une intrigante acharnée ou comme une femme blessée, malheureusement éconduite et à qui le ressentiment n’inspirerait qu'amertume et désir de revanche. "Il n'est resté d'elle que la Nemesis vengeresse », écrit ainsi Paul Gautier dans sa préface (p. III), tandis qu'Henri Guillemin évoque « le petit cri de stupeur calculée et d'émerveillement et d'émotion engageante qu' elle pousse, le 6 décembre 1797 [...] lorsqu'elle est tout à coup en présence du 'héros' » (p. 24). Comploteuse malveillante ou admiratrice blessée dans son amour-propre, Mme de Staël reste donc toujours une femme, et une femme passionnée, dans son face-à-face avec l'homme impérial. Cette interprétation partiale, et à bien des égards misogyne, n’a évidemment pas son équivalent dans les travaux staëliens. Envisagée en termes historiques, autrement dit comme un événement dont la réalité exige le dépouillement des archives de police et des témoignages de l'époque, la relation entre Mme de Staël et Napoléon doit au travail scientifique de Simone Balayé et Norman King notamment, entre 1974 et $1990^{7}$, d'avoir progressivement substitué la vérité aux fantasmes. Leur exploration rigoureuse des documents relatifs à la censure de De l'Allemagne - les exemplaires du premier tirage du traité staëlien, explicitement consacré à " la patrie de la pensée ${ }^{8}$ ", sont saisis avant d'être détruits, chez le libraire Nicolle le 28 septembre 1810 sur ordre de Rovigo - rend à l'épopée staëlienne sa réalité, et du même geste sa complexité. Le topos de la victime larmoyante s'y lézarde en effet au profit de l'image, beaucoup plus trouble, d'un auteur parfaitement lucide sur les risques encourus par son projet éditorial, mais pour

\footnotetext{
4 Voir sur cette question la récente synthèse de Léonard BURNAND, « $\mathrm{M}^{\mathrm{me}}$ de Staël et Napoléon : mythe et réalités d'un duel », dans Germaine de Staël, retour d'exil, Zoé : Carouge, 2015, p. 45-77.

5 Paul GAUTIER, Madame de Staël et Napoléon, Paris : Plon, 1903.

6 Henri GUILLEMIN, Madame de Staël et Napoléon ou Germaine et le caïd ingrat, Paris : éditions du Panorama, 1966.

Voir notamment " Madame de Staël et le gouvernement impérial en 1810, le dossier de la suppression de De l'Allemagne ", dans Cahier staëliens, n 19, 1974, p. 3-77 ; "L'écrivain et le pouvoir ", chap. 3 de Lumières et liberté, Paris : Klincksieck, 1979, p. 75-119 ; et avec Norman KING, " Madame de Staël et les polices françaises sous la Révolution et l'Empire ", dans Cahiers staëliens, n 44, 1992-1993, p. 3-153.

8 Germaine de STAËL, De l'Allemagne, 1813, réed. Paris : Hachette, 1958, t. I, p. 12.
} 
qui la transgression constitue non seulement une nécessité, mais aussi une jouissance. En témoigne la saisissante revendication de Mme de Staël elle-même dans les Considérations sur la Révolution française : évoquant, au chapitre vir de la 4e partie, intitulé « Le dernier ouvrage de M. Necker sous le consulat de Bonaparte ", les Dernières vues de politique et de finance publié par l'ancien ministre en 1802 et qui lui valent, outre une réception calomnieuse, le blâme officiel du consul Lebrun, Staël revendique le droit de dissocier sa propre dissidence de celle de son père :

"On conseillait ensuite à $M$. Necker de ne plus se mêler de politique, et de s'en remettre au Premier consul, seul capable de bien gouverner la France : ainsi les despotes trouvent toujours les penseurs de trop dans les affaires. Le consul finissait en déclarant que moi, fille de M. Necker, je serais exilée de Paris, précisément à cause des Dernières vues de politique et de finance publiées par mon père. J'ai mérité depuis, je l'espère, cet exil aussi pour moi-même?." "

"Mériter [s]on exil aussi pour [s]oi-même ": un tel programme s'accorde difficilement avec le mythe de la « Nemesis vengeresse » ou de l'admiratrice éconduite.

\section{Retour sur l'affaire De l'Allemagne}

Il explique pourtant l'étrange affaire de De l'Allemagne, première entorse problématique à la légende de la victime suppliciée face au chef tout-puissant. Le dépouillement des archives de police, entre 1809 et 1812, joint à la lecture attentive de la Correspondance, révèle en effet, chez Mme de Staël, une recherche quasi délibérée de l'interdiction. Alors que Napoléon incarne traditionnellement, dans ce dossier, la répression aveugle et despotique, les études de Simone Balayé révèlent au contraire la responsabilité partagée des protagonistes dans l'épisode le plus célèbre de la librairie impériale : l'examen du rapport établi par les censeurs sur De l'Allemagne, le 26 septembre 1810, prouve en effet qu'ils autorisent, sous réserve que quelques passages soient modifiés, la publication du livre. Cette décision s'explique en grande partie par des motifs stratégiques : le pouvoir redoute, à juste titre, que De l'Allemagne ne gagne un éclat supplémentaire s'il paraît à l'étranger et l'histoire leur donnera raison : la première édition « libre », à Londres, chez John Murray en septembre 1813, auréole le traité d'un irrésistible parfum de scandale et de subversion. Attendu, interdit et désiré, De l'Allemagne, grâce au duc de Rovigo, électrise ses lecteurs et constitue l'événement éditorial de la fin de l'Empire. Mme de Staël aurait-elle donc sciemment anticipé les vertus de cette répression en provoquant elle-même Napoléon, prenant le risque d'annuler le rapport favorable des censeurs? La lettre qu'elle lui envoie, datée du 25 septembre 1810, 
le suggère, qui, sous couvert de demander l'assouplissement de l'exil, réitère le farouche attachement de Mme de Staël à son indépendance :

"Tant de gens demandent à Votre Majesté des avantages réels de toute espèce, pourquoi rougiraisje de lui demander l'amitié, la poésie, la musique, les tableaux, toute cette existence idéale dont je puis jouir sans m'écarter de la soumission que je dois au monarque de la France ${ }^{10}$ ?"

L'éloge de l'humanité, de la beauté et du monde intérieur explicite chez l'opposante l'existence d'un territoire inaccessible aux chantages politiques, aux forces armées et au despotisme. Travestie en supplique, la lettre de Mme de Staël surenchérit donc dans la provocation et l'interdiction de De l'Allemagne, loin d'émaner d'abord du pouvoir, sanctionne a posteriori l'audace d'une femme rappelant à l'Empereur, au moment même où ses censeurs examinent le travail de six années, sa liberté.

L'affaire De l'Allemagne, examinée scientifiquement, c'est-à-dire archives à l'appui, invalide ainsi la légende de la victime pathétique. Étape cruciale du conflit qui oppose Mme de Staël à Napoléon, elle révèle un surprenant partage des responsabilités et, chez la prétendue femme éplorée, un scénario transgressif mûrement réfléchi. Ce jeu dangereux témoigne, chez chacun des protagonistes, de caractères passionnés. Aucune trace, ici, de l'abdication définie par les Réflexions sur le suicide comme l'idéal philosophique du sujet libre. Mme de Staël réussit pourtant, au cœur d'un des épisodes les plus tourmentés de sa destinée, à convertir le négatif de l'opposition en principe dynamique : le contrôle de l'imprimerie, loin de brider sa création, la décuple au contraire et nul doute que la profondeur métaphysique de De l'Allemagne, sous le signe de la disparition et de l'exploration des labyrinthes intérieurs, n’ait gagné une audace supplémentaire à l'épreuve de cette destruction. La préface en témoigne, qui associe la tentation du néant qui traverse le texte à l'anéantissement des volumes et à ce que le texte appelle, fasciné, " leur transformation en un carton parfaitement blanc sur lequel aucune trace de la raison humaine n'est restée ${ }^{11} »$. Sublimer l'interdit constitue ainsi la première étape de Staël vers l'abdication : s'affranchir des passions qui obscurcissent la relation à ses adversaires et à « ceux qui ne nous ressemblent pas », pour reprendre la formule des Réflexions sur le suicide, suppose d'abord de ne plus souffrir de leurs persécutions. Napoléon, s’il reste un adversaire dans cette affaire, voit en revanche son rôle stratégiquement infléchi : Mme de Staël lui doit, paradoxalement, une part de la réussite de son texte et ce virage inaugure la dernière page, la plus méconnue, mais aussi la plus surprenante, de leurs relations.

10 Lettre à Napoléon du 24-25 septembre 1810, dans Correspondance générale, éd. Béatrice JASINSKI et Othenin d'HAUSSONVILLE, Genève : Slatkine, 2008, t. VII, p. 260.

11 De l'Allemagne, p. 4. 


\section{Dix années d'exil ou l'illusion polémique}

Elle s'appuie cette fois non plus sur les archives de police, mais sur l'œuvre elle-même. Mme de Staël consacre en effet deux grands textes à sa relation avec l'Empereur : Dix années d'exill', recueil de souvenirs commencé en 1810 et qui reste inachevé, et les Considérations sur la Révolution française, publiées de manière posthume en 1818, mais dont Mme de Staël commence la rédaction vers 1796. Ces deux œuvres n'occupent curieusement qu'une place mineure dans les études consacrées aux deux personnages. Mme de Staël y souligne pourtant l'étroite relation qui unit sa propre trajectoire à celle de Napoléon :

"L'empereur Napoléon, dont le caractère se montre tout entier dans chaque trait de sa vie, m'a persécutée avec un soin minutieux, avec une activité toujours croissante, avec une rudesse inflexible, et mes rapports avec lui ont servi à me le faire connaître longtemps avant que l'Europe ềt compris le mot de cette énigme et lorsquelle se laissait dévorer par le sphinx parce qu'elle n'avait pas su le deviner ${ }^{13}$."

Formulée à l'ouverture des Dix années d'exil, cette déclaration explicite l'importance, à la fois biographique et historiographique, des témoignages staëliens. Comprendre ce qui se joue entre ces deux figures ne saurait faire l'économie d'un récit qui mêle aussi étroitement la vie de l'héroïne à celle de son persécuteur. Dix années d'exil livre cependant, sur la figure de l'Empereur, un tableau à charge. Saturé d'anecdotes blessantes, de détails malveillants et d'attaques personnelles - " De tout temps, sa taille a été ignoble, sa gaieté vulgaire, sa politesse gauche [...], sa façon d'être grossière et rude, surtout avec les femmes ", lit-on notamment dans les premières pages (p. 49) -, il témoigne d'une subjectivité qui explique sans doute, pour une part, le mythe d'une Mme de Staël vengeresse, haineuse et incapable de livrer un juste témoignage sur son ennemi. Dix années d'exil déroge en effet spectaculairement à "l'abdication de la personnalité ». Blessée et oppressée par les douleurs de l'exil, Staël y succombe, pour la première et unique fois de sa carrière, à la méchanceté, à la satire et au racisme - on ne compte pas les attaques contre " le Corse qui ne voulait se servir de la grande nation que pour opprimer l'Europe " (p. 123) ni les rappels que « la fille de M. Necker était plus française que lui » (p. 385). Cette écriture au vitriol ne relève cependant pas, chez Mme de Staël, d'un choix assumé. Imputable à l'inachèvement du texte, la noirceur du témoignage signe moins le triomphe de la haine que l'état intermédiaire d'un manuscrit publié sans recul ni révision finale de l'auteur. C'est du moins l'explication avancée par Auguste de Staël dans la préface dont il accompagne, au tome XV des Euvres complètes de sa mère,

12 Dix années d'exil, 1812, rééd. Simone BALAYÉ et Mariella Vianello BONIFACIO, Paris : Fayard, 1996.

13 Id., p. 45. 
éditées chez Treuttel et Würtz, un texte d'autant plus problématique qu'il se révèle pour la première fois au public en 1821, soit l'année même de la mort de Napoléon :

"Si l'on compare les Dix années d'exil avec les Considérations sur la Révolution française, on trouvera peut-être que le règne de Napoléon est jugé dans le premier de ces écrits avec plus de sévérité que dans l'autre, et qu'il y est attaqué avec une éloquence qui n'est pas toujours exempte d'amertume. Cette différence est facile à expliquer: l'un de ces ouvrages a été écrit après la chute du despote, avec le calme et l'impartialité d'un historien; l'autre a été inspiré par un sentiment courageux de résistance à la tyrannie; et quand ma mère l'a composé, le pouvoir impérial était à son apogée $e^{14}$."

Auguste établit ici une distinction de taille : si Dix années d'exil succombe, partiellement, à la tentation de "l'amertume ", la faute en incombe à l'urgence d'une chronique rédigée en direct de l'événement, sans médiation ni possible élaboration d'une perspective historique. L'hypothèse, loin d'être la justification subjective d'un fils nécessairement juge et partie des mémoires de sa mère, se confirme à la lecture des nombreuses notes préparatoires rédigées par Staël elle-même : elle y précise, notamment dans sa correspondance, que le manuscrit des Dix années d'exil n'aurait jamais dû voir le jour sous sa forme, inachevée, de tableau partisan et de souvenirs intimes. Programmé pour neutraliser les impressions subjectives et effacer jusqu'à la moindre trace d'anecdotes particulières, le texte final, intitulé De l'exil, n'aurait conservé du moi qu'une trace seule capable d'incarner l'échelle universelle de la réflexion. Mme de Staël s'en explique précisément dans une lettre à Claude Hochet datée du 5 mai 1812 :

"Il y a neuf ans que je souffre et deux années que je ne vis plus. C'est un état assez singulier et je suis tentée de faire un ouvrage intitulé De l'exil, qui serait, je crois, rempli d'observations assez nouvelles sur le cour humain ; je m'y mettrais moi-même comme épisode ${ }^{15}$."

S'explicite ici, outre le projet d'un traité, le caractère transitoire d'un document écrit sur le vif et où résonne une souffrance d'autant plus impudique, aux yeux de Mme de Staël, qu'elle surgit sans voile et contrevient non seulement à son éthique d'écrivain, mais au projet même de raconter une vie : "De même que tous les visages prêtent à la caricature, écrit-elle en effet dans Dix années d'exil (p. 155), l'existence de chaque individu a toujours quelque chose de ridicule si l'on veut l'examiner sans mélange d'affection ou d'enthousiasme. " La vie de l'Empereur lui-même, à lire un tel programme, exige donc, à qui prétend la retracer, une juste distance et non la plume acide de la satire. Si Staël n'a pas le temps

14 Dix années d'exil, Euvres complètes de Madame la baronne de Staël publiées par son fils, Paris : Treuttel et Würtz, 1821, t. xv, p. XIII-XIV.

15 Correspondance générale, t. VII, p. 582. 
d'atteindre cet équilibre dans Dix années d'exil, il s'impose en revanche, et de manière saisissante, dans les Considérations sur la Révolution française.

\section{Les Considérations ou le regard apaisé sur l'Empire}

La lecture de ce monument, à la fois tombeau du père trop aimé et premier regard surplombant sur les années 1789-1814, révèle en effet bien des surprises qui déjouent, sans conteste, les stéréotypes de l'historiographie classique. Simone Balayé l'avait pressenti, qui annonçait déjà, en 1974, la nécessité d'une réinterprétation du dossier de ce qu'elle appelait alors "l'écrivain et le pouvoir ${ }^{16}$ " : " L'histoire de $M^{\text {me }}$ de Staël, note-t-elle, devra, un jour, être entièrement réécrite, et notamment l'histoire de ses rapports avec l'Empereur ${ }^{17}$. "Si elle s'était alors concentrée sur les archives de l'affaire De l'Allemagne, la relecture des Considérations, elle, restait à faire. Mme de Staël y atteint de fait, au crépuscule de sa carrière, une impartialité et une "équité philosophique »- pour reprendre la formule programme des Réflexions sur le suicide - qui l'autorisent à jeter un regard objectif, voire fraternel sur Napoléon. Elle s'en explique elle-même à différentes reprises en dénonçant d'abord, de manière générale, l'aveuglement stérile de l'esprit de parti :

"La grande erreur des hommes passionnés en politique, c'est d'attribuer tous les genres de vices et de bassesses à leurs adversaires. Il faut savoir apprécier à quelques égards ceux qu'on hait, et ceux même qu'on méprise; car nul homme, et surtout nulle masse d'hommes n'a jamais entièrement abdiqué tout sentiment moral ${ }^{18}$."

Adressée aux royalistes coupables, selon elle, d'avoir refusé l'aide de La Fayette en 1792, la formule, " apprécier ceux qu'on hait » désigne l'idéal de neutralité qui doit engager et les protagonistes et les auteurs à privilégier, jusque dans leur relation des faits, un regard lucide, capable de surmonter les préjugés de corps et les intérêts personnels. Cette gageure semble a priori moins difficile lorsqu’il s'agit, comme c'est le cas dans ce chapitre, de retracer les circonstances, déjà presque lointaines, de la chute de la royauté. Mme de Staël n'a pourtant pas peur de l'étendre, beaucoup plus courageusement, à l'histoire récente et au tableau même de l'Empire:

"Dans les diverses observations que je viens de rassembler sur Bonaparte, je n'ai point approché de sa vie privée que j'ignore, et qui en concerne pas les intérêts de la France. Je n'ai pas dit un fait douteux sur son histoire; car les calomnies quion lui a prodiguées me semblent plus viles encore

\footnotetext{
16 La formule donne son titre au chapitre 4 de son ouvrage, Lumières et liberté, Paris: Klincksieck, 1979, p. 75-119.

17 Simone BALAYÉ, "Madame de Staël et le gouvernement impérial en 1810, le dossier de la suppression de De l'Allemagne ", dans Cahiers staëliens, n 19, 1974, p. 4.

18 Considérations..., p. 277.
} 
que les adulations dont il fut l'objet. Je me flatte de l'avoir jugé comme tous les hommes publics doivent l'être: d'après ce quils ont fait pour la prospérité, les lumières et la morale des nations. Les persécutions que Bonaparte m’a fait éprouver n'ont pas, je puis l'attester, exercé d'influence sur mon opinion ${ }^{19}$."

Concluant son récit des Cent-Jours, cette revendication de neutralité surprend d'autant plus qu'elle intervient sous la plume de la principale opposante - " Je fus la première femme que Bonaparte exila ", rappelle Mme de Staël au chapitre intitulé « De l'exil » (p. 386) - et en direct ou presque de l'événement. L'« Avertissement " placé par Auguste en tête de la première édition des Considérations, en 1818, précise en effet que "Madame de Staël avait achevé dès les premiers jours de 1816 la composition de l'ouvrage " (t. XII, p. 4). L'abdication de Napoléon, si elle réussit à dissocier la chronique des événements des lésions personnelles de l'auteur, ne bénéficie donc d'aucun recul ni d'aucune distance temporelle. Comment Mme de Staël a-t-elle alors réussi, si près des faits, à mettre à distance ses souvenirs et ses deuils ? Ce programme dessine-t-il un horizon idéal ou la couleur réelle de son tableau de la chute de l'Empire?

La réponse à ces questions surgit au cœur de l'avant-dernière partie des Considérations. Quatre chapitres y sont en effet consacrés aux derniers jours de Napoléon. Ce volume conséquent offre un premier indice, sous la plume de Mme de Staël, non seulement de l'importance accordée à l'événement, mais aussi de sa volonté d'en retracer de la manière la plus exhaustive les circonstances. Il est évidemment facile de soupçonner aussi, dans une tout autre perspective, le désir de revanche de la femme exilée qui trouverait enfin, dans ce récit détaillé, l'occasion d'une vengeance, voire d'une délectation au spectacle de l'ancien ennemi enfin à terre. Rien de tel pourtant n'anime son récit des Cent-Jours. Mme de Staël ne s'y contente pas de rendre explicitement hommage à son adversaire ; elle admire aussi la résistance, voire la richesse dramatique d'une fin de partie riche en effets et en rebondissements inattendus. La structure de son récit témoigne, à ce titre, de l'intérêt à la fois stratégique et esthétique d'une séquence conçue par Staël comme un diptyque : fidèle au rythme de l'événement lui-même, orchestré à la manière d'un coup de théâtre au point que l'historien Francis Démier parle, dans son récent ouvrage, de "l'aventure des Cent-Jours ${ }^{20}$ ", le tableau des Considérations déroule en deux étapes, respectivement situées dans la quatrième et la cinquième partie : le départ, puis le retour par surprise de l'Empereur. Cette partition, outre qu'elle restitue la stupeur des contemporains et plus particulièrement celle de Mme de Staël, enfin de retour à Paris après douze années d'exil, le 12 mai 1814, souligne aussi la spectaculaire résistance d'un ennemi capable de déjouer le scénario, trop vite écrit, de sa disparition. Mme de Staël ne s'y trompe pas, qui restitue aux Cent-Jours leur dimension de bravade et de geste sublime :

19 Id., p. 435.

20 Francis DÉMIER, La France de la Restauration (1814-1830). L'Impossible retour du passé, Paris : Gallimard, 2012 , p. 86-152. 
"Je ne me livrerai point, comme on ne se l'est que trop permis, à des déclamations de tout genre contre Napoléon. Il a fait ce quil était naturel de faire, en essayant de regagner le trône quil avait perdu, et son voyage de Cannes à Paris est une des plus grandes conceptions de l'audace qúon puisse citer dans l'histoire ${ }^{21}$. "

Surprenante sous la plume de celle qui est directement menacée par la résurrection de l'Empereur - Mme de Staël n'a de fait d'autre choix que de quitter à nouveau Paris pour se réfugier à Coppet le 10 mars 1815 -, cette admiration atteste une abdication effective des passions personnelles. Mme de Staël, actrice malgré elle d'un événement qui relance la longue histoire d'un conflit qui dure depuis 1803 , ne réussit pas seulement à s'oublier dans une fresque envisagée à la hauteur des nations et des énergies qui traversent l'Europe ; elle trouve aussi la force morale d'envisager objectivement son adversaire en rendant hommage aux qualités dont il fait preuve, à commencer par le courage - « On ne saurait se permettre d'accuser Bonaparte de manquer de bravoure dans cette circonstance », précise-t-elle (p. 503) -, l'autorité - «Il était très habile dans l'art de commander une armée » (p. 501) - et ce que Mme de Staël appelle "l'héritage de sa terrible puissance " (p. 436). Toutes nourrissent une énergie hors du commun, et qui empruntent ses ressorts au sublime :

"Cet homme a vécu pour donner au monde la leçon de morale la plus frappante, la plus sublime dont les peuples aient jamais été témoins ${ }^{22}$."

Les Considérations, loin de céder à la tentation du réquisitoire, inscrivent donc au contraire Napoléon au panthéon des grandes figures du temps. Du génie, il possède en effet l'audace transgressive qui le pousse à l'exploit - «Ébranler l'Europe par son retour » (p. 503) constitue une prouesse qui séduit la plupart des contemporains - et le conduit parfois aux portes de l'extrême.

Ce recul des limites permet à Mme de Staël de franchir une étape supplémentaire dans son écriture neutre. À l'assourdissement des griefs personnels et des désirs de vengeance s’ajoute ainsi, dans les Considérations, le portrait quasi fraternel du héros. Il commence par la peinture empathique de la seconde abdication. Au moment où la force, d'abord du côté de Napoléon dont même les adieux gardent dignité et grandeur, bascule dans le camp des opposants qui cherchent à travestir l'Empereur en roi constitutionnel, Mme de Staël déplore, paradoxalement, la triste déchéance d'un militaire soudain privé de sa scène d'élection :

"La terreur quil inspirait, la puissance qui résultait de cette terreur n'existaient plus ; c'était un ours muselé qu'on entendait murmurer encore, mais que ses conducteurs faisaient danser à leur façon. Au lieu d'obliger à parler constitution pendant des heures entières, il fallait qu'il fût

\footnotetext{
1 Considérations..., p. 496.

2 Id., p. 503.
} 
en campagne quatre jours après son arrivée à Paris [...]. Il fallait qu’il soulevât les passions des Italiens et des Polonais [...]; enfin, quil prît la liberté comme arme et non comme entrave ${ }^{23}$."

L'analyse renverse ici la perspective sur les Cent-Jours : Napoléon, sous la plume de Staël, s'y rend moins coupable d'un coup de force que d'une trahison de la nature "intensiviste " de son caractère. Héritée des Lumières ${ }^{24}$, cette notion désigne la recherche, nécessaire aux âmes passionnées, de l'existence morale la plus extrême et la plus exaltante. Rien de plus oppressant, pour ceux que consument leurs facultés, que l'ennui et le spectre de l'inactivité. Ce diagnostic réoriente par conséquent l'interprétation du débarquement au Golfe-Juan : l'Empereur, si l'on en croit la version staëlienne de l'événement, se rend moins coupable d'un coup de force qu'il ne suit l'inclination naturelle d'un tempérament chez lui frénétique. Les Considérations mêlent ici subrepticement au regard de l'historien l'analyse morale d'un cas d'autant plus fascinant pour Staël qu'elle y reconnaitt, en miroir, le spectre de sa mélancolie :

"Cette vieille Europe ménnuie, disait Napoléon avant de partir pour la Russie. En effet, il ne rencontrait plus d'obstacles à ses volontés nulle part, et l'inquiétude de son caractère avait besoin d'un aliment nouveau. Peut-être aussi la force et la clarté de son jugement saltérèrent-elles, quand les hommes et les choses plièrent tellement devant lui, qu'il n'eut plus besoin d'exercer sa pensée sur aucune des difficultés de la vie. Il y a dans le pouvoir sans bornes une sorte de vertige qui saisit le génie comme la sottise, et les perd également l'un et l'autre25."

La peur du vide et l'angoisse des intervalles, si elle impose au conquérant la recherche effrénée d'une extension vouée par principe à l'échec, résonne aussi chez l'écrivain poussé, sa vie entière, par les rythmes cyclothymiques de la passion. Mme de Stäl ne se contente donc pas d'éclairer sous un jour objectif les énergies qui motivent l'aventure exceptionnelle de l'Empire ; elle rend aussi à son chef un potentiel fantasmatique qu'elle éprouve par résonance et dont la disparition menace d'exténuation son œuvre elle-même. Là réside l'ultime paradoxe des Considérations qu'elles déplorent en effet, avec la chute de Napoléon, la disparition d'un sujet d'inspiration sans précédent :

"Maintenant ce n'est plus lui seul qui occupera l'histoire dont nous voulons esquisser le tableau, et notre malheureuse France va de nouveau reparaître, après quinze ans pendant lesquels on n'avait entendu parler que de l'Empereur et de son armée ${ }^{26}$."

Qui va désormais remplir la scène ? Cette inquiétude explique aussi, sur le plan esthétique, la singulière longueur d'un chapitre dont Mme de Staël tient à exploiter jusqu'au bout la matière. Tout se passe, à découvrir le détail et la précision de ses interprétations, comme s’il fallait à tout prix reculer le

\footnotetext{
23 Id., p. 499.

24 Voir Michel DELON, L'Idée d'énergie au tournant des Lumières : 1770-1820, Paris : Presses universitaires de France, 1988.

25 Considérations, p. 426.

26 Id., p. 435.
} 
moment de faire ses adieux à un personnage, à tous les sens du terme, aussi complexe pour les historiens qu'il inspire naturellement la fiction. "Mais on ne peut se taire sur Bonaparte ", avoue-t-elle en effet (p. 436), explicitant le déficit, pour les écrivains comme pour les moralistes, à voir disparaître une aussi fascinante énigme.

\section{La lecture anthropologique}

Napoléon ne représente en effet, pour Mme de Staël, ni un bourreau, ni un coupable. Si sa restitution des faits réussit à éviter les vapeurs toxiques du ressentiment, elle réussit aussi, dans un ultime tour de force, à déplacer la question de la responsabilité historique de l'Empereur. À ceux qui chercheraient trop facilement des explications psychologiques ou politiques à l'instauration d'un régime où Mme de Staël identifie la perversion despotique des appétits, elle oppose le mystère d'une âme insondable et dont les Considérations elles-mêmes ne sauraient épuiser le mystère :

"On peut penser diversement sur son génie et sur ses qualités; il y a quelque chose d'énigmatique dans cet homme qui prolonge la curiosité. Chacun le peint sous d'autres couleurs, et chacun peut avoir raison du point de vue qu'il choisit; qui voudrait concentrer son portrait en peu de mots, n'en donnerait qu'une fausse idée. Pour arriver à quelque ensemble, il faut suivre diverses routes: c'est un labyrinthe qui a un fil, l'égoïsme ${ }^{27}$. "

La métaphore du « labyrinthe » explicite la profondeur énigmatique d'une figure non seulement fascinante pour les écrivains, mais que sa complexité préserve aussi de toute interprétation partiale ou prématurée. Qui est Napoléon ? Présentée comme insoluble, sinon au prix d'une exploration condamnée à l'errance, la question permet à Mme de Staël, stratégiquement, de réserver à l'Empereur une place à part dans la chasse aux responsabilités qui menace de s'emparer de la France restaurée. L'enjeu, pour elle, est double : esthétique - l'observatrice des passions identifiant dans son sujet une profondeur irréductible aux explications simples - et politique. La France, pour Mme de Staël, doit assumer sa propre responsabilité dans une déviation de principes dont la faute est moins imputable à un seul individu qu’à la nation dans son ensemble. Cette analyse, ultime étape du projet étonnamment lucide des Considérations, inspire le titre polémique du chapitre qui ouvre la sixième et dernière partie : "Les Français sont-ils faits pour être libres? " (p. 509). Mme de Staël y examine, plutôt que les travers de l'Empereur, les conditions sociales, politiques et morales qui ont au contraire autorisé l'instauration d'un mal dont elle tient à souligner la dimension collective. Là réside en effet, à lire les Considérations, la clé de voûte de l'Empire. Sa logique conquérante résulte moins de la folie d'un homme que de la vacuité

27 Id., p. 504. 
d'une nation rendue inerte par le choc révolutionnaire et où ne subsistent plus, en guise d'intérêt, que l'espoir des victoires et la dépendance des âmes contraintes de s'en remettre, pour vibrer, au calendrier impérial. La tyrannie, si elle a pu plonger ses racines en France, y a donc rencontré, sous la plume de Mme de Staël, un terreau fertile, s'appelât-il " ambition » (p. 435), " jacobinisme militaire » (p. 500) ou égoïsme, "le goût de l'argent et des honneurs » (p. 511), comme elle le nomme elle-même, ayant exercé son éternelle attraction.

L'analyse staëlienne établit ici un partage polémique des responsabilités : dissociant l'aliénation du seul chef, elle rend la nation organiquement complice et modalise de facto le réquisitoire contre l'Empereur. Le mal dictatorial n'a pas une origine unique, pas plus que la Terreur à qui Mme de Staël avait déjà appliqué, quelques années plus tôt, la même méthode. Plusieurs similitudes relient en effet, à suivre son raisonnement, le règne de Robespierre à celui de Napoléon. Politiquement d'abord, puisque 1793 représente, dans l'optique libérale, une trahison de l'élan révolutionnaire comparable à celle que l'Empire, devenu monarchie absolue, impose à l'héritage républicain et aux principes de la Révolution. Moralement ensuite, puisque le même phénomène se reproduit, qui voit une nation - ce que Mme de Staël appelle " une masse d'hommes " - s'aliéner elle-même en consentant à perdre son jugement pour confier tous les pouvoirs à un seul homme. Une fois la raison recouvrée et ce régime d'exception renversé, la tentation est grande de déplacer la faute sur le seul chef : sa culpabilité ne permet-elle pas, en effet, d'épargner au pays tout entier l'examen de sa propre part d'ombre ? Face à cette mauvaise foi ou à ce déni collectif, Staël adopte donc, en 1795 comme en 1816, la même stratégie : celle qui consiste à désolidariser le prétendu tyran de la nation pour lui substituer l'hypothèse, bien plus troublante, d'une complicité de tous dans l'émergence du despotisme. Cette lecture morale de l'histoire, identifiant sous la divergence des contextes une structure passionnelle qui pousse toujours le sujet à rechercher, paradoxalement, son propre aveuglement ${ }^{28}$, innocente le chef, ou plutôt refuse de le considérer comme une exception criminelle ni comme un monstre. Robespierre bénéficie le premier, sous la plume de Mme de Staël, de cette neutralisation. Elle intervient dans les Réflexions sur la paix intérieure, publiées en 1795 et qui partagent déjà, avec les Considérations, une analyse presque immédiate de l'événement : "Il faut qu'un jour l'histoire détaillée de cet homme soit soumise à l'examen des moralistes. [...] On y verra que la secte démagogique existait très indépendamment de Robespierre [...], que de certains signes, de certains tics quion a examinés dans lui, lui sont communs avec tous les hommes de ce temps-là, un tressaillement dans les nerfs, ces convulsions dans les mains, ces mouvements de tigre dans la manière de s'agiter à la tribune, de se porter à droite et à gauche comme les animaux

28 Voir Stéphanie GENAND, «La femme eunuque : Germaine de Staël et la lecture négative du despotisme », dans Cahiers staëliens, n65, 2015 , p. 89-113. 
dans leur cage, tous ces détails curieux qui montrent le passage de la nature humaine à celle des bêtes féroces, sont absolument pareils dans la plupart des hommes, cités pour leur cruauté29. "

Dissocier le prétendu tyran de la tyrannie et souligner son appartenance au genre humain, dont il est le représentant exalté plus que l'incompréhensible anomalie, là réside la méthode staëlienne. Pardelà la différence des circonstances, elle préconise toujours de s'abstraire des jugements personnels pour privilégier, face au surgissement du mal, le scalpel critique de la raison.

Cette anatomie ou courage de la distinction porte chez Mme de Staël un nom : elle s'appelle la considération. Ce terme crucial, avant d'être choisi comme titre du grand ouvre testamentaire qui conclut sa carrière, désigne l'écriture impersonnelle de l'histoire. Faculté de faire disparaître ses propres intérêts au profit d'une lecture non seulement lucide, mais aussi universelle de l'événement, la considération préserve l'histoire du jugement et de l'esprit de parti qui menace consubstantiellement l'interprétation des faits. Elle se traduit, chez Mme de Staël, par la mise en concurrence de deux régimes de représentation : l'immédiat, qui va reconstituer le détail des circonstances et rendre à chaque épisode de l'histoire ses spécificités; la profondeur, capable de retrouver, sous l'écume des événements, le jeu des passions et la tendance, incompréhensible, de l'individu à suspendre l'exercice de son jugement. Cette double échelle relativise systématiquement la responsabilité des chefs et explique que Mme de Staël absolve, après Robespierre, Bonaparte. Elle détermine aussi, sous sa plume, l'évocation relative de l'Empire. Alors que Napoléon y apparaît comme un génie hors pair, écartelé entre l'ivresse de la puissance et la mélancolie, les quinze années de son règne rejoignent aussi l'âge mythique d'une histoire de l'humanité régulièrement traversée par la violence.

Les armées impériales, ainsi envisagées à l'échelle des passions plus qu'à celles des faits, inaugurent moins un nouveau chapitre de la fresque du temps qu'elles ne réveillent les appétits souterrains dont toute civilisation se nourrit et dont les ambitions conquérantes de Bonaparte ne sont qu'un des symptômes. Cette interprétation mythique ou, pourrait-on dire, archaïque de l'Empire, à la fois histoire contemporaine et réveil des ressorts obscurs de l'âme humaine, explique chez Mme de Staël le puissant réseau métaphorique qu'il inspire. Napoléon, d'abord affranchi des noirceurs de l'ennemi, devient ensuite, sous sa plume, une figure de légende, qu'il s’agisse du "Sphinx ", pour reprendre une image récurrente des Dix années d'exil et des Considérations, qui évoquent notamment un «Sphinx d'un nouveau genre, [et] c'était contre celui qui devinait ses énigmes que se tournait sa fureur » (p. 382), ou de la « horde » régulièrement invoquée pour désigner ses troupes armées : «Il n’y a de bonheur que là où l'on n'est pas soumis à la grande horde ", écrit Mme de Staël à Caroline von Humboldt dans une

29 Réflexions sur la paix intérieure, 1795, rééd. Lucien JAUME, Euvres complètes de Madame de Staël, III-1, p. 136. 
lettre à paraitre du 27 novembre 1812, faisant écho au parallèle, développé dans Dix années d'exil, entre les guerriers tartares mis en scène dans un spectacle à Saint-Pétersbourg et les armées corses :

"Je vis une tragédie russe intitulée Dimitri Donskoj, dont le sujet était la délivrance des Russes lorsquills repoussèrent les Tartares par-delà Kazan. Le prince de Smolensk, le prince de Tver paraissaient dans l'ancien costume des boyards et l'armée tartare s'appelait la Horde dorée. Ce nom pourrait bien convenir à l'armée corse $e^{30}$."

Horde, sphinx, énigme : les images offrent à Mme de Staël un réseau imaginaire mieux à même de donner à voir, sous la réalité historique de l'Empire, les forces mythiques et par-là même intemporelles qui le structurent.

Qu'ajouter, en conclusion, pour refermer cette relecture de la relation entre Germaine de Staël et Napoléon ? Rappeler l'importance des textes et de la lecture des œuvres, dont l'examen attentif révèle seul une complexité de discours irréductible aux simplifications partisanes. On pourrait bien sûr objecter qu'il est difficile de croire Mme de Staël sur parole lorsqu'elle retrace elle-même des événements dont elle a si profondément souffert. Force est pourtant de constater que l'abdication ne constitue pas seulement, chez elle, un idéal. Elle est au cour des Considérations, véritable bréviaire philosophique qui prouve, en pleines négociations politiques et alors que l'Europe s'apprête à instaurer un nouvel ordre au congrès de Vienne, la possibilité de jeter sur le présent, même le plus brûlant, un regard juste parce que dépassionné. L'œuvre staëlienne indique ainsi, à qui entreprend de la lire, la voie à suivre pour une interprétation lucide du jeu et des passions politiques. Elle apprend les vertus de l'universel, le poison des égoïsmes, le danger des aveuglements partisans et rappelle la nécessité, au cœur des périodes les plus sombres de l'histoire, de ne jamais substituer à la raison la peur de l'autre ni la protection de ses seuls intérêts. De 1816 à 2016, deux cents ans se sont ainsi écoulés, qui ne retirent pourtant rien au défi de la considération. Lire Germaine de Stäl nous rappelle, aujourd'hui encore, les vertus de l'abdication.

30 Dix années d'exil, p. 305. 